\title{
An Uplink Resource Allocation Algorithm for OFDM and FBMC Based Cognitive Radio Systems
}

\author{
Musbah Shaat and Faouzi Bader \\ Centre Tecnològic de Telecomunicacions de Catalunya (CTTC) \\ Parc Mediterrani de la Tecnología, Av. Carl Friedrich Gauss 7, 08860 , Castelldefels-Barcelona, Spain. \\ Phone: +34 93 6452911, Fax: +34 93 6452900, Email:\{musbah.shaat,faouzi.bader\}@cttc.es
}

\begin{abstract}
This paper presents an uplink radio resource allocation algorithm for multicarrier based cognitive radio systems. The algorithm allocates the subcarriers to the users in the first step. In the second step, the power is allocated to these subcarriers in order to maximize the total data rate of the system without causing excessive interference to the primary user. The performance of the proposed algorithm is investigated using computer simulations to prove that it achieves a very good performance in comparison with optimal and outperforms other existing algorithms. Moreover, the throughput of Orthogonal frequency division multiplexing (OFDM) and filter bank multicarrier system (FBMC) based cognitive radio systems are compared to show the efficiency of using FBMC in future cognitive radio systems.
\end{abstract}

\section{INTRODUCTION}

The cognitive radio (CR) technology aims to increase the spectrum utilization by allowing a group of unlicensed users [referred to as secondary users (SUs)] to use the licensed frequency channels (spectrum holes) without causing a harmful interference to the licensed users [referred to as primary users (PUs)] and thus implement efficient reuse of the licensed channels [1].

Due to its flexibility in allocating different resources among different users as well as its ability to fill the spectrum holes left by the PU's, multicarrier communication (MC) systems have been considered as a promising candidate for CR systems [2]. In [3], the mutual interference between PU and SU was studied. The mutual interference depends on the transmitted power as well as the spectral distance between PU and SU. Orthogonal frequency division multiplexing (OFDM) based $\mathrm{CR}$ system suffers from high interference to the PU's due to large sidelobes of its filter frequency response. Moreover, the insertion of the cyclic prefix (CP) in each OFDM symbol decreases the system capacity. Filter bank multicarrier system (FBMC) with the offset quadrature amplitude modulation (OQAM) can achieve smaller intersymbol interference (ISI) and intercarrier interference (ICI) without using the CP by utilizing well designed pulse shapes that satisfy the perfect reconstruction conditions. Moreover, the problem of the spectral leakage can be solved by minimizing the sidelobes of each

This work was partially supported by the European ICT-2008-211887 project PHYDYAS, COST Action IC0902 and Generalitat de Catalunya under grant 2009-SGR-940. subcarrier which leads to high efficiency (in terms of spectrum and interference) [4], [5].

The problem of uplink resource allocation has been already studied in classical (non-cognitive) MC systems [6], [7] and references therein. In [6], the authors proposed a greedy subcarrier allocation algorithm based on marginal rate function and iterative waterfilling power allocation algorithm. This algorithm is developed in [7] to consider the fairness among the different users. The algorithms used in classical MC systems may be not valid for CR context due to the existence of the mutual interference between the PUs and SUs.

Although the downlink MC based CR system has been addressed well recently (see, e.g. [8]-[11]), there is a few existed research on the subcarrier and power allocation in uplink multicarrier based CR systems. In Our work in [10][11], an algorithm for resource allocation in downlink MC based CR systems was presented whereby the subcarrier is allocated to the user with the maximum signal to noise (SNR) ratio (optimal in downlink) and then Lagrangian method is used to find the power allocation. The allocation of the subcarriers to the user with maximum SNR is not efficient in uplink due to the per user power constraints in addition to the different interference gains between the different SUs and the primary system which motivates proposing an efficient subcarrier allocation algorithm in uplink MC based CR systems. Wang et al. proposed in [12] an algorithm to allocate resources in the uplink OFDMA based CR systems under the per-subcarrier power constraints. The subcarriers are allocated initially to the users with the best channel quality and then adjusted according to the different user's waterfilling levels. The algorithm has limited performance comparing with the optimal solution.

In this paper, we propose an efficient resource allocation algorithm in multicarrier based CR systems. The objective is to maximize the total SUs data rate while limiting the interference introduced to the primary system. The proposed resource allocation is divided into two steps. The subcarrier to user assignment is first performed based on the channel quality, amount of interference imposed to the primary system and the increment in the total data rate. In the second step, the power is allocated to the different users under the per-user total power and interference constraints. The efficiency of the proposed algorithm will be investigated in OFDM and FBMC based 


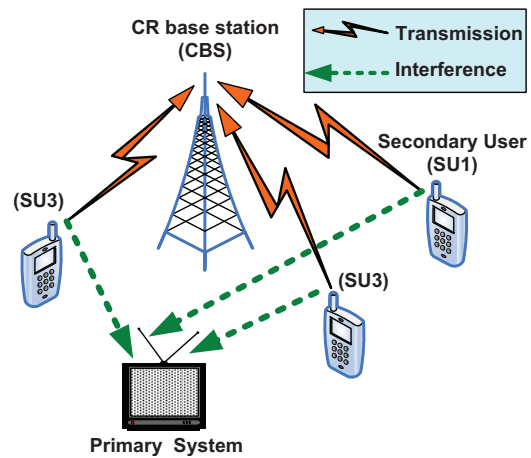

Fig. 1. Uplink Cognitive Radio Network.

CR systems. Simulation results demonstrate that the proposed algorithm achieves a good performance and outperforms the algorithm presented by Wang et. al in [12].

\section{System Model AND Problem Formulation}

In this paper, the uplink scenario will be considered. As shown in Fig. 1, the CR system coexists with the primary system in the same geographical location. The SUs transmit to their cognitive base station (CBS) and cause interference to the primary system. Moreover, the primary system base station interferes with the SUs. The CR system's frequency spectrum is divided into $N$ subcarriers each having a $\Delta f$ bandwidth. The side by side frequency distribution of the primary system band and the CR bands will be assumed (see Fig. 2). The frequency band $B$ has been occupied by the primary system (PU band) while the other bands represent the inactive primary system bands (CR bands). It is assumed that the CR system can use the inactive primary bands provided that the total interference introduced to the primary system does not exceed $I_{t h}$ where $I_{t h}=T_{t h} B$ denotes the maximum interference power that can be tolerated by the primary system and $T_{t h}$ is the interference temperature limit for the primary system.

OFDM and FBMC based CR systems will be assumed. The OFDM symbol is formed by taking the inverse discrete Fourier transform (IDFT) to a set of complex input symbols and then adding the $\mathrm{CP}$ while the OQAM/FBMC symbols can be formed by modulating each subcarrier with a staggered QAM. The basic idea is to transmit real-valued symbols instead of transmitting complex valued ones. Due to this time staggering of the in-phase and quadrature components of the symbols, orthogonality is achieved between adjacent subcarriers. The modulator and the demodulator are implemented using the synthesis and analysis filter banks. The filters in the synthesis and analysis filter bank are obtained by frequency shifts of a single prototype filter. When considering block diagrams, the polyphase network (PPN) block replaces the device for $\mathrm{CP}$ insertion/removing used in OFDM terminals as shown in Fig. 3. The FFT is common to both OFDM and FBMC, which is an important aspect for the compatibility issues.

Assume that $\Phi_{i}$ is the power spectrum density (PSD) of the $i^{t h}$ subcarrier. The expression of the PSD depends on the used

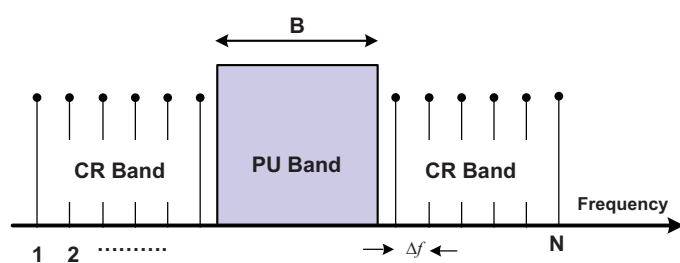

Fig. 2. Frequency distribution of the primary and cognitive bands.



Fig. 3. Block diagram of OFDM and FBMC systems.

multicarrier technique. If an OFDM based CR is assumed, the PSD of the $i^{t h}$ subcarrier can be written as

$$
\Phi_{i}(f)=P_{i}\left|G_{i}(f)\right|^{2}
$$

where $P_{i}$ is the total transmit power emitted by the $i^{\text {th }}$ subcarrier and $G_{i}(f)$ is the Fourier transform of the used pulse shape $g_{T}$. Assuming a rectangular pulse with length $T_{s}=N+C$ where $N$ is the number of subcarriers (IDFT size) and $C$ is the length of the $\mathrm{CP}, G_{i}(f)$ can be expressed as follows

$$
\left|G_{i}(f)\right|^{2}=T_{s}+2 \sum_{r=1}^{T_{s}-1}\left(T_{s}-r\right) \cos (2 \pi f r)
$$

If FBMC based CR system is assumed, the PSD of the $i^{\text {th }}$ subcarrier can be written as

$$
\Phi_{i}(f)=P_{i}\left|H_{i}(f)\right|^{2}
$$

where $\left|H_{i}(f)\right|$ is the frequency response of the prototype filter with coefficients $h[n]$ with $n=0, \cdots, W-1$, where $W=K N$ and $K$ is the length of each polyphase components (overlapping factor). Assuming that the prototype coefficients have even symmetry around the $\left(\frac{K N}{2}\right)^{t h}$ coefficient, and the first coefficient is zero [4], we get

$\left|H_{i}(f)\right|=h[W / 2]+2 \sum_{n=1}^{\frac{W}{2}-1} h[(W / 2)-n] \cos (2 \pi n(f-i / N))$

The mutual interference introduced by the $i^{\text {th }}$ subcarrier to the PU band, $I_{i}\left(d_{i}, P_{i}\right)$, is the integration of the PSD of the 
$i^{\text {th }}$ subcarrier across the PU band, $B$, and can be expressed as [3]

$$
I_{i}\left(d_{i}, P_{i}\right)=\int_{d i-B / 2}^{d i+B / 2}\left|g_{i}\right|^{2} \Phi_{i}(f) d f=P_{i} \Omega_{i}
$$

where $d_{i}$ is the spectral distance between the $i^{\text {th }}$ subcarrier and the PU band and $g_{i}$ is the $i^{t h}$ subcarrier fading gain from the SU to the primary system. $\Omega_{i}$ denotes the interference factor of the $i^{t h}$ subcarrier. The interference power introduced by the primary system's signal into the band of the $i^{\text {th }}$ subcarrier is [3]

$$
J_{i}\left(d_{i}, P_{P U}\right)=\int_{d i-\Delta f / 2}^{d i+\Delta f / 2}\left|y_{i}\right|^{2} \psi\left(e^{j \omega}\right) d \omega
$$

where $\psi\left(e^{j \omega}\right)$ is the power spectrum density of the primary system's signal and $y_{i}$ is the channel gain between the $i^{t h}$ subcarrier and the primary system signal. It will be assumed that all the instantaneous fading gains are perfectly known at the CBS. The channel gains between the SUs and the CBS can be obtained practically by the classical channel estimation techniques while the channel gains between the CBS/SUs and the PUs can be obtained by the CBS/SUs via, e.g., estimating the received signal power from each primary terminal when it transmits, under the assumptions of pre-knowledge on the primary transmit power levels and the channel reciprocity [13]. The SUs will assumed to be perfectly synchronized and no frequency synchronization is considered between the PUs and SUs. Based on this information, the CBS assigns the subcarrier and power to each SU through a reliable low-rate signaling channel. Let $\omega_{i, m}$ to be the subcarrier allocation indicator, i.e. $\omega_{i, m}=1$ if and only if the $i^{t h}$ subcarrier is allocated to $m^{\text {th }}$ user. It is assumed that each subcarrier can be used for transmission to at most one user at any given time. The transmission rate of the $i^{\text {th }}$ subcarrier, $R_{i}$, with the transmit power $P_{i, m}$ can be evaluated using the Shannon capacity formula and is given by

$$
R_{i}\left(P_{i, m}, h_{i, m}\right)=\Delta f \log _{2}\left(1+\frac{P_{i, m}\left|h_{i, m}\right|^{2}}{\sigma_{i}^{2}}\right)
$$

where $h_{i, m}$ is the $i^{t h}$ subcarrier fading gain from the CBS to the $m^{\text {th }}$ SU. $\sigma_{i}^{2}=\sigma_{A W G N}^{2}+J_{i}$ where $\sigma_{A W G N}^{2}$ is the variance of the additive white Gaussian noise (AWGN) and $J_{i}$ is the interference introduced by the primary system's signal into the $i^{\text {th }}$ subcarrier and can be evaluated using (6). The noise variance is assumed to be constant for all the users and subcarriers.

Our objective is to maximize the total capacity of the CR system subject to the instantaneous interference introduced to the primary system and the per-user transmit power constraints. Therefore, the optimization problem can be formu- lated as follows

$$
\begin{array}{lll}
P 1: & \max _{P_{i, m}, \omega_{i, m}} \sum_{m=1}^{M} \sum_{i=1}^{N} \omega_{i, m} R_{i}\left(P_{i, m}, h_{i, m}\right) \\
\text { s.t. } & \sum_{m=1}^{M} \sum_{i=1}^{N} \omega_{i, m} P_{i, m} \Omega_{i, m} \leq I_{t h} \\
& \sum_{i=1}^{N} P_{i, m} \leq \overline{P_{m}}, \quad \forall m \\
& P_{i, m} \geq 0, \quad \forall i, m \\
\omega_{i, m} \in\{0,1\}, \quad \forall i, m \\
\sum_{m=1}^{M} \omega_{i, m} \leq 1, \quad \forall i
\end{array}
$$

where $N$ denotes the total number of subcarriers while $M$ denotes the number of SUs. $I_{t h}$ is the interference threshold prescribed by the primary system, $\Omega_{i, m}$ is the interference factor given by (5) and $\overline{P_{m}}$ is the $m^{t h}$ user total power budget. The last constraint ensures that any given subcarrier can be allocated to at most one user at any given time.

The optimization problem $P 1$ is a NP-hard optimization problem in which achieving the optimal solution needs unacceptable computational complexity. In order to reduce the computational complexity, the resource allocation problem can be solved in two steps. In the first step, the subcarriers are assigned to the users and then, the power is allocated for these subcarriers in the second step. In the sequel, the proposed subcarrier to user assignment scheme is introduced and then the power allocation is discussed.

\section{Proposed Subcarrier and Power Allocation ALGORITHM}

The optimal downlink subcarriers to users allocation scheme in MC systems is achieved by allocating each subcarrier to the user with the maximum signal to noise ratio (SNR). This scheme of subcarrier allocation is not efficient in uplink case due to the per-user power constraints. Moreover, the interference introduced to the primary system by each SU should be considered in CR context which makes the schemes used in classical multicarrier systems not valid. For this reason, a heuristic subcarrier allocation algorithm is presented. The proposed algorithm tries to assign the subcarriers to the different SUs considering not only their channel quality and per-user power constraints but also the interference that will be induced to the primary system. It is well known that the subcarriers that are located close to the PU band are introducing more interference to the primary system than the far subcarriers and hence, as the subcarrier becomes more close to the PU band, the interference to the primary system will approximately guide the subcarrier allocation process while the channel quality has more effect on the allocation of the distant subcarriers.

The scheme initially assumes that the interference introduced to the primary system is uniform among the different subcarriers which means that every subcarrier is allowed to introduce the same amount of interference to the primary system, i.e. $I_{U n i f o r m}=\frac{I_{t h}}{N}$. By using (5), the maximum 
power that can be allocated to the $i^{t h}$ subcarrier when it is allocated to the $m^{\text {th }} \mathrm{SU}$ is

$$
P_{i, m}^{U n i}=\frac{I_{U n i f o r m}}{\Omega_{i, m}}
$$

Let $\mathcal{U}$ to be the set of unassigned subcarriers. We define the sets $\mathcal{A}_{m}$ and $\mathcal{B}_{m}$ to include all the subcarriers already allocated to the $m^{t h} \mathrm{SU}$ according to maximum power, i.e $P_{i, m}^{U n i}$, and average power respectively. The average power means that the left power for the $m^{t h}$ user $\left(\overline{P_{m}}-\sum_{i \in \mathcal{A}_{m}} P_{i, m}^{U n i}\right)$ is divided equally among the subcarriers in the set $\mathcal{B}_{m}$. The algorithm begins by the allocation of the subcarriers that are located spectrally next to the PU band, i.e. subcarriers that have more interference to the PU, and moving towards the distant ones. The assigning procedures of a particular subcarrier $i^{*} \in \mathcal{U}$ are as follows

1) $\forall m$, Evaluate $P_{\text {Test }}=\frac{\overline{\overline{P_{m}}}-\sum_{r \in \mathcal{A}_{m}} P_{r, m}^{U n i}}{\left|\mathcal{B}_{m}\right|+1}$

if $P_{\text {Test }} \geq P_{i^{*}, m}^{U n i}$

let $\mathcal{A}^{\prime}{ }_{m}=\mathcal{A}_{m} \cup\left\{i^{*}\right\}$ and $\mathcal{B}^{\prime}{ }_{m}=\mathcal{B}_{m}$

else let $\mathcal{B}^{\prime}{ }_{m}=\mathcal{B}_{m} \cup\left\{i^{*}\right\}$ and $\mathcal{A}_{m}^{\prime}=\mathcal{A}_{m}$.

$|\mathcal{X}|$ means the cardinality of the set $\mathcal{X}$.

2) Compute the amount of increment $\Delta_{m}$ in the data rate when the subcarrier $\left\{i^{*}\right\}$ is assigned to $m^{\text {th }} \mathrm{SU}$, i.e,

$$
\Delta_{m}=R_{m}^{\text {new }}-R_{m}^{\text {old }}
$$

where

$$
\begin{aligned}
R_{m}^{\text {new }}= & \sum_{i \in \mathcal{B}_{m}^{\prime} m} R_{i}\left(\frac{\overline{P_{m}}-\sum_{r \in \mathcal{A}_{m}^{\prime}} P_{r, m}^{U n i}}{\left|\mathcal{B}_{m}^{\prime}\right|}, h_{i, m}\right)+ \\
& \sum_{i \in \mathcal{A}_{m}^{\prime}} R_{i}\left(P_{i, m}^{U n i}, h_{i, m}\right) \\
R_{m}^{\text {old }}= & \sum_{n \in \mathcal{B}_{m}} R_{i}\left(\frac{\overline{P_{m}}-\sum_{r \in \mathcal{A}_{m}} P_{r, m}^{U n i}}{\left|\mathcal{B}_{m}\right|}, h_{i, m}\right)+ \\
& \sum_{i \in \mathcal{A}_{m}} R_{i}\left(P_{i, m}^{U n i}, h_{i, m}\right)
\end{aligned}
$$

and $R_{i}\left(P_{i, m}, h_{i, m}\right)$ is evaluated using (7).

3) Find $m^{*}$ satisfying $m^{*}=\arg \max _{m}\left(\Delta_{m}\right)$, set $\omega_{i^{*}, m^{*}}=1$, and update the sets $\mathcal{A}_{m^{*}}=\mathcal{A}_{m^{*}}^{\prime}$ and $\mathcal{B}_{m^{*}}=\mathcal{B}^{\prime}{ }_{m^{*}}$.

4) Remove the subcarrier $i^{*}$ form the set $\mathcal{U}$ and repeat the above procedures until the set $\mathcal{U}$ is empty.

The importance of the first step in the proposed algorithm is in the consideration of the limitation that will be introduced to any subcarrier assignment due the interference constraint. Generally speaking, the subcarriers that will introduce high interference to the primary system will have a low transmitting power which will reduce the total data rate even that they have a good channel quality. Hence, the first step helps in classifying the subcarriers according to their interference to the primary system. Afterwards, the increment of the data rate due to allocation of a particular subcarrier to different SUs is evaluated and the subcarrier is allocated to the SU with maximum data rate increment. The scheme is repeated until the allocation of all subcarriers. Note that the final set of allocated subcarriers to $m^{\text {th }} \mathrm{SU}$ is $\mathcal{N}_{m}=\mathcal{A}_{m} \cup \mathcal{B}_{m}$.

Once the subcarriers are allocated to the users, the multiuser system can be viewed virtually as a single user multicarrier system. The values of the subcarrier indicators, i.e. $\omega_{i, m}$, are already known from the subcarriers to users assignment step. For simplicity of notation, the problem $P 1$ is reformulated as follows

$$
\begin{array}{ll}
P 2: & \max _{P_{i, m}} \sum_{i=1}^{N} R_{i}\left(P_{i, m}, h_{i, m}\right) \\
\text { s.t. } & \sum_{i=1}^{N} P_{i, m} \Omega_{i, m} \leq I_{t h} \\
& \sum_{i \in \mathcal{N}_{m}} P_{i, m} \leq \overline{P_{m}} \quad \forall m \\
& P_{i, m} \geq 0 \quad \forall i
\end{array}
$$

where $m$ in $P_{i, m}, h_{i, m}$ and $\Omega_{i, m}$ refers to the user who's already got the subcarrier $i$, i.e. $\omega_{i, m}=1$. $\mathcal{N}_{m}$ denotes the set of subcarriers allocated to the $m^{t h}$ SU. Remark that having too much power comparing to the interference constraint will lead to an interference-only optimization problem while having high interference constraint in relative with the total power will lead to a non-cognitive, i.e. classical, resource allocation problem. The problem $P 2$ is a convex optimization problem. The Lagrangian can be written as

$$
\begin{array}{r}
G=-\sum_{i=1}^{N} R_{i}\left(P_{i, m}^{*}, h_{i, m}\right)+\alpha\left(\sum_{i=1}^{N} P_{i, m}^{*} \Omega_{i, m}-I_{t h}\right) \\
+\sum_{m=1}^{M} \beta_{m}\left(\sum_{i \in \mathcal{N}_{m}} P_{i, m}^{*}-\overline{P_{m}}\right)-\sum_{i=1}^{N} P_{i, m}^{*} \mu_{i}
\end{array}
$$

where $\alpha, \mu_{i}$, and $\beta_{m}$ are the non-negative Lagrange multipliers. The Karush-Kuhn-Tucker (KKT) conditions can be written as follows

$$
\begin{aligned}
& P_{i, m}^{*} \geq 0 ; \quad \alpha \geq 0 ; \quad \beta_{m} \geq 0 ; \quad \mu_{i} \geq 0 ; \quad \mu_{i} P_{i, m}^{*}=0 \\
& \alpha\left(\sum_{i=1}^{N} P_{i, m}^{*} \Omega_{i, m}-I_{t h}\right)=0 \\
& \beta_{m}\left(\sum_{i \in \mathcal{N}_{m}} P_{i, m}^{*}-\overline{P_{m}}\right)=0, \forall m \\
& \frac{\partial G}{\partial P_{i, m}^{*}}=\frac{-1}{\frac{\sigma_{i}^{2}}{\left|h_{i, m}\right|^{2}}+P_{i, m}^{*}}+\alpha \Omega_{i, m}+\sum_{m=1}^{M} \beta_{m}-\mu_{i}=0
\end{aligned}
$$

and also the solution should satisfy the per-user power and total interference constraints. Rearranging the last condition in (12) we get

$$
P_{i, m}^{*}=\frac{1}{\alpha \Omega_{i, m}+\sum_{m=1}^{M} \beta_{m}-\mu_{i}}-\frac{\sigma_{i}^{2}}{\left|h_{i, m}\right|^{2}}
$$

From (12) and (13), the optimal solution can be written as follows

$$
P_{i, m}^{*}=\left[\frac{1}{\alpha \Omega_{i, m}+\sum_{m=1}^{M} \beta_{m}}-\frac{\sigma_{i}^{2}}{\left|h_{i, m}\right|^{2}}\right]^{+}
$$

where $[x]^{+}=\max (0, x)$. These multipliers can be found numerically using ellipsoid or interior point method with a polynomial time complexity [14]. 
TABLE I

COMPLEXITY COMPARISON

\begin{tabular}{|l|l|}
\hline Algorithm & Complexity \\
\hline Optimal (Exhaustive Enumeration) & $\mathcal{O}\left(N^{3} M^{N}\right)$ \\
\hline Wang & $\in\left[\mathcal{O}\left(N^{2} M\right), \mathcal{O}\left(N^{3} M\right)\right]$ \\
\hline Classical & $\mathcal{O}(N M)+\mathcal{O}\left(N^{3}\right)$ \\
\hline Proposed & $\mathcal{O}(N M)+\mathcal{O}\left(N^{3}\right)$ \\
\hline
\end{tabular}

\section{Complexity Analysis And Simulations Results}

The simulations are performed under the scenario given in Fig.1. The values of $\Delta f$, and $\sigma_{i}^{2}$ are assumed to be 0.3125 $\mathrm{MHz}$ and $10^{-6}$ respectively. The channel gains $h$ and $g$ are outcomes of independent Rayleigh distributed random variables with mean equal to 1 . All the results have been averaged over 1000 iterations. OFDM and FBMC based cognitive radio systems are evaluated. The OFDM system is assumed to have a $6.67 \%$ of its symbol time as CP. For FBMC system, the prototype coefficients are assumed to be equal to PHYDYAS coefficients with overlapping factor $K=4$ [15]. For the purpose of performance comparison, the following algorithms are considered:

1) Optimal: the subcarriers are allocated by exhaustive enumeration while the power is allocated using (14).

2) Wang: the method proposed in [12] is used. The interference constraint is converted into per-subcarrier power constraints using (9).

3) Classical: the subcarriers are allocated according to the scheme used in non-cognitive MC systems [7], while the power is allocated using (14).

The exhaustive enumeration scheme needs to iterate $M^{N}$ times to exhaust all the cases, and its complexity of $\mathcal{O}\left(N^{3} M^{N}\right)$ is very hard to afford. Moreover, the Wang's algorithm in [12] has a complexity larger than $\mathcal{O}\left(N^{2} M\right)$ and lower than $\mathcal{O}\left(N^{3} M\right)$. Both the Classical and the Proposed algorithms need to perform $M$ function evaluations to assign one subcarrier to one user. Hence, the computational complexity of the subcarrier to user allocation algorithm in the proposed and classical algorithms is $\mathcal{O}(N M)$. Additionally, both the classical and proposed algorithms need to solve (14) to find the optimal power allocation with a complexity $\mathcal{O}\left(N^{3}\right)$. Therefore, the computational complexity of the classical and the proposed algorithm is $\mathcal{O}(N M)+\mathcal{O}\left(N^{3}\right)$. Table.I summarizes the computational complexity of the different algorithms.

Fig. 4 plots the average capacity of a CR system with $M=2$ SUs versus the interference threshold when the number of subcarriers is $N=8$, the per-user power budget $\overline{P_{m}}=1 \mathrm{~m}$ Watt and $B=2.5 \mathrm{MHz}$. The proposed algorithm achieves a good performance in comparison with optimal and outperforms the other algorithms. Moreover,the capacity of FBMC based CR system is higher than that of OFDM based one because the sidelobes in FBMC's PSD are smaller than that in OFDM which introduces less interference to the PU's. Moreover, the inserted CP in OFDM based CR systems reduces the total capacity of the system. For the rest of the results, the optimal solution will not be simulated due

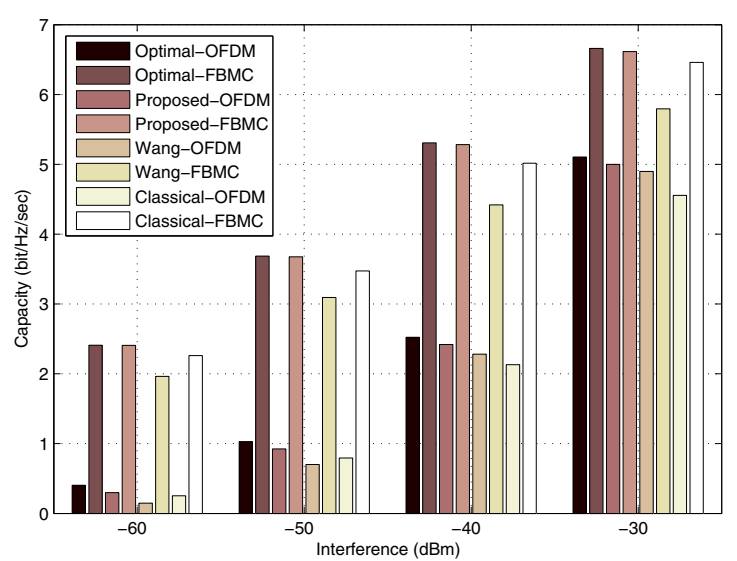

Fig. 4. Achieved capacity vs interference threshold.

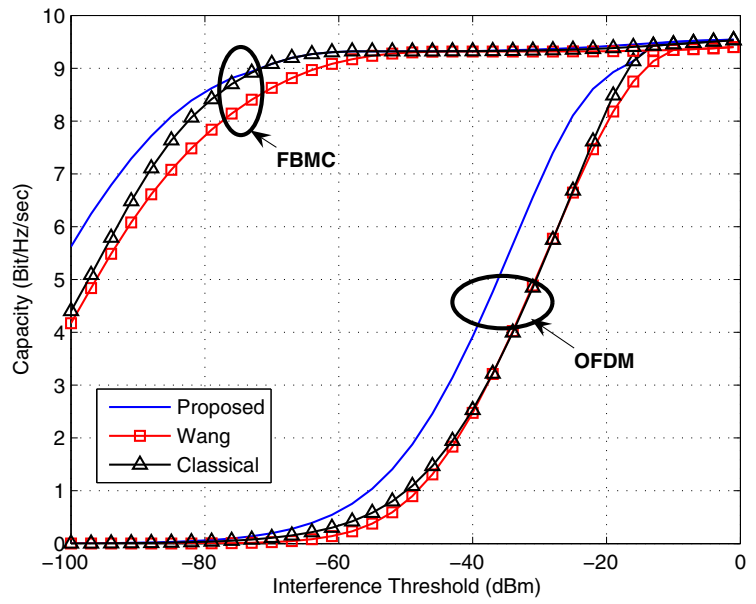

Fig. 5. Achieved capacity vs allowed interference threshold.

to its high computational complexity when the numbers of subcarriers and users are increased.

Fig. 5 plots the average capacity versus the interference threshold when the number of subcarriers is $N=64$, the number of SUs is $M=10$, the per-user power budget is $\overline{P_{m}}=1 \mathrm{~m}$ Watt and $B=10 \mathrm{MHz}$. It can be observed that as the interference threshold increases, the capacity increases since each SU is allowed to have more flexibility in allocating more power on its subcarriers. The performance of the proposed algorithm outperforms other algorithms. Moreover, the gap between the different algorithms decreases with the interference threshold as the CR system becomes more closer to the classical (non-cognitive) system. The capacity of FBMC based CR system is higher than that of OFDM.

In the case of OFDM system, the interference has a high effect on the system performance where the efficiency of the proposed algorithm appears. Moreover, the inefficiency of the Classical algorithm is shown when the interference constraint affects the optimization problem. In FBMC systems, the difference between the Classical and the proposed is very small. The reason behind that is in the low interference induced by the FBMC system to the primary system which makes 


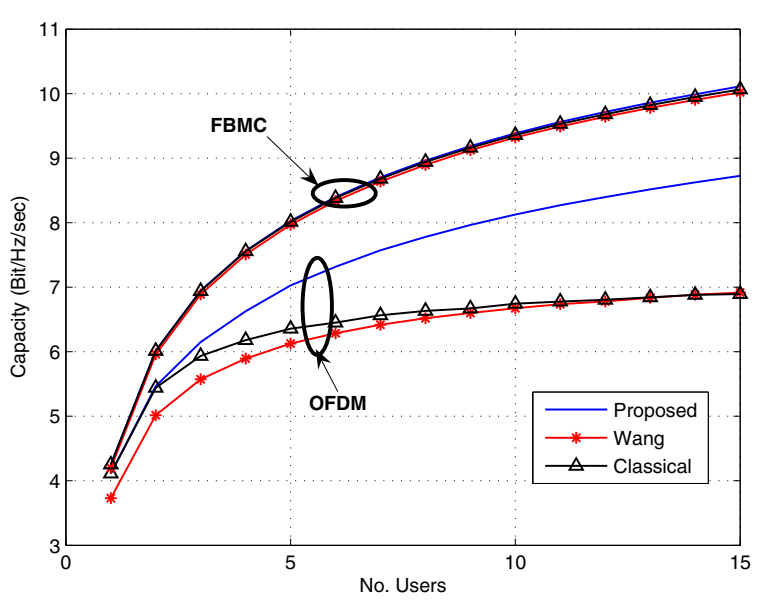

Fig. 6. Achieved capacity vs No. of SUs.

the CR system very close to the non-conative one. In FBMC CR system with an extremely small interference threshold (or with high power budget), the proposed algorithm will be useful and achieves more capacity than the classical one as in the region below $-70 \mathrm{dBm}$ in Fig. 5 for example. Since the Classical and the proposed algorithm apply the same power allocation algorithm, its clear that the capacity increase of the proposed algorithm over the Classical one results from the subcarrier allocation step. In addition to the subcarrier allocation, the conversion of the total interference constraint into several per-subcarrier maximum power constraints, choosing the minimum waterfilling level, and the assumptions made to decrease the computational complexity affect the performance of Wang's algorithm.

Fig. 6 shows the average capacity versus the number of SUs when the number of subcarriers is $N=64$, the interference thresholds is $-25 \mathrm{dBm}$, the per-user power is $\overline{P_{m}}=1 \mathrm{mWatt}$ and $B=10 \mathrm{MHz}$. The capacity increases with the number of users due to the multiuser diversity. In OFDM based CR system, the lower the number of SUs, the smaller the difference between the proposed and Classical algorithm. This is because the number of subcarriers that will be allocated to each user will increase which reduces the amount of power that will be allocated to each subcarrier and consequently the amount of interference imposed to the primary system which makes the CR system acts relatively as a non-cognitive system. FBMC based CR system has more capacity than the OFDM based one and the different algorithms have approximately the same capacity because the interference introduced to the primary system by the FBMC based CR system is less than the prescribed threshold and hence, works virtually like the non-cognitive schemes.

From the presented results, one can notes that when the interference constraint affects the optimization the problem (like the OFDM case), the proposed algorithm enhances the total data rate and outperforms the other algorithms. Additionally, due to its small interference to the PU, the throughput of FBMC systems is more than the OFDM ones and the classical ways of subcarrier allocation can be used (depending on the values of power and interference constraints) which recommends the FBMC as a candidate for the CR applications

\section{CONCLUSION}

In this paper, we proposed an efficient resource allocation algorithm for uplink in MC based CR networks. The allocation process is separated into two steps. In the first step, the subcarriers are allocated to the users according to their channel quality as well as the interference that they may introduce to the primary system. In the second step, the per-user power budget is distributed among the subcarriers so that the total system capacity is maximized without causing excessive interference to the primary system. It is shown that the proposed algorithm outperforms Wang's algorithm and achieves a very good performance. Moreover, simulation results prove that the FBMC based CR systems have more capacity than OFDM based ones. The obtained results contribute in recommending the use of FBMC physical layer in the future cognitive radio systems.

\section{REFERENCES}

[1] S. Haykin, "Cognitive radio: brain-empowered wireless communications," IEEE Journal on Selected Areas in Communications, pp. 201220, Feb. 2005.

[2] T. Weiss and F. K. Jondral, "Spectrum pooling: An innovative strategy for the enhancement of spectrum efficiency," IEEE Communications Magazine, vol. 42, pp. S8 - S14, March 2004.

[3] T. Weiss and J. Hillenbrand, "Mutual interference in OFDM-based spectrum pooling systems," in Vehicular Technology Conference (VTC'04Spring), vol. 4, May 2004.

[4] B. Farhang-Boroujeny and R. Kempter, "Multicarrier communication techniques for spectrum sensing and communication in cognitive radios," IEEE Commun. Mag. (Special Issue on Cognitive Radios for Dynamic Spectrum Access), vol. 48, no. 4, pp. 80-85, Apr. 2008.

[5] H. Zhang, D. Le Ruyet, and M. Terre, "On spectral efficiency analysis between OFDM/OQAM and OFDM based CR networks," in Vehicular Technology Conference (VTC'09-Spring), Barcelona-Spain, 2009.

[6] K. Kim, Y. Han, and S.-L. Kim, "Joint subcarrier and power allocation in uplink OFDMA systems," IEEE Communications Letters, vol. 9, no. 6, pp. 526-528, Jun 2005.

[7] L. Gao and S. Cui, "Efficient subcarrier, power, and rate allocation with fairness consideration for OFDMA uplink," IEEE Transactions on Wireless Communications, vol. 7, no. 5, pp. 1507-1511, May 2008.

[8] G. Bansal, M. J. Hossain, and V. K. Bhargava, "Optimal and suboptimal power allocation schemes for OFDM-based cognitive radio systems," IEEE Transactions on Wireless Communications, vol. 7, no. 11, pp. 4710-4718, November 2008

[9] Y. Zhang and C. Leung, "Resource allocation in an OFDM-based cognitive radio system," IEEE Transactions on Communications, vol. 57, no. 7, pp. $1928-1931$, july 2009.

[10] M. Shaat and F. Bader, "Computationally efficient power allocation algorithm in multicarrier-based cognitive radio networks: OFDM and FBMC systems," EURASIP Journal on Advances in Signal Processing, vol. 2010, Article ID 528378, 13 pages ,2010.

[11] M. Shaat and F. Bader, "A two-Step resource allocation algorithm in multicarrier based cognitive radio system," in IEEE Wireless Communications and Networking Conference (IEEE WCNC'10), SydneyAustralia, April 2010.

[12] W. Wang, W. Wang, Q. Lu, and T. Peng, "An uplink resource allocation scheme for OFDMA-based cognitive radio networks," International Journal of Communication Systems, vol. 22, no. 5, pp. 603-623, 2009.

[13] R. Zhang, S. Cui, and Y.-C. Liang, "On ergodic sum capacity of fading cognitive multiple-access and broadcast channels," IEEE Transactions on Information Theory, vol. 55, no. 11, pp. 5161-5178, nov. 2009.

[14] S. Boyd and L. Vandenberghe, Convex optimization. Cambridge, U.K.: Cambridge Univ. Press, 2004.

[15] "PHYDYAS-Physical layer for dynamic spectrum access and cognitive radio," Project website: www.ict-phydyas.org. 